\title{
Polyelectrolyte Complexes Based on Chitosan and Natural Polymers
}

\author{
Alef Mustafa \\ Umf Carol Davila Bucharest, Faculty of Pharmacy, Bucharest, Romania \\ Aneta Tomescu \\ "Ovidius" University of Constanţa, Faculty of Medicine, Constanta, Romania \\ Emin Cadar \\ Umf Carol Davila Bucharest, Faculty of Pharmacy, Bucharest, Romania \\ Melat Cherim \\ Umf Carol Davila Bucharest, Faculty of Pharmacy, Bucharest, Romania \\ Rodica Sîrbu \\ "Ovidius" University of Constanţa, Faculty of Pharmacy, Constanta, Romania
}

\begin{abstract}
For many years chitosan has been the subject of interest for its use in different medical fields due to its appealing properties such as biocompatibility, biodegradability, low toxicity and relatively low production cost from abundant natural sources. Chitosan is positively charged at low pH values, so it is spontaneously associated with negatively charged polyions in solution to form polyelectrolyte complexes. These chitosan based polyelectrolyte complexes exhibit favourable physicochemical properties with preservation of chitosan's biocompatible characteristics. These chitosan based complexes are a good candidate for excipient materials for the design of different types of dosage forms. The aim of this review is to describe polyelectrolyte complexes of chitosan with selected natural polyanions and also to indicate some of the factors that influence the formation and stability of these formed complexes.
\end{abstract}

Keywords: chitosan; polyelectrolyte complex; natural polymers

\section{Introduction}

Chitosan is a combination of a series of polymers that are deacetylated derivatives of chitin,a natural polysaccharide,and presents different degrees of deacetylation and molecular weights. It consists of deacetylated units of $\beta-1,4$-linked glucosamine and acetylated units of $\mathrm{N}$-acetyl-D-glusoamine (Figure 1). Chitosan presents typical degrees of deacetylation between 70 and $95 \%$ and molecular weights between 10 and $1,000 \mathrm{kDa}[1,2]$. It was reported that highly refined grades of chitosan have been used in pharmaceutical formulations as a release-controlling agent [3].

It was shown that the cationic amino groups on the $\mathrm{C} 2$ position of the repeating glucopyranose units of chitosan can interact electrostatically with the anionic groups (usually carboxylic acid groups) of other polyions to form polyelectrolyte complexes. Many different polyanions from natural origin (e.g. pectin, alginate, carrageenan, xanthan gum, carboxymethyl cellulose, chondroitin sulphate, dextran sulphate, hyaluronic acid) or synthetic origin (e.g., poly (acrylic acid)), polyphosphoric acid, poly (L-lactide) have been used to form polyelectrolyte complexes with chitosan in order to provide the required physicochemical properties for the design of specific pharmaceutical formulations [4].

Thoughout the years chitosan complexes have been used in a wide range of pharmaceutical applications such as complexes formed between chitosan and anionic polymers for use as biosensors, scaffolds in tissue engineering, for wastewater treatment and for drug delivery in different forms $[5,6]$. 
Most of the investigated and studied polycomplexes that involve chitosan are those formed with other polysaccharides, which are divided into natural polysaccharides (including phytopolysaccharides, zoopolysaccharides and microorganism polysaccharides) and synthetic polysaccharides [7]

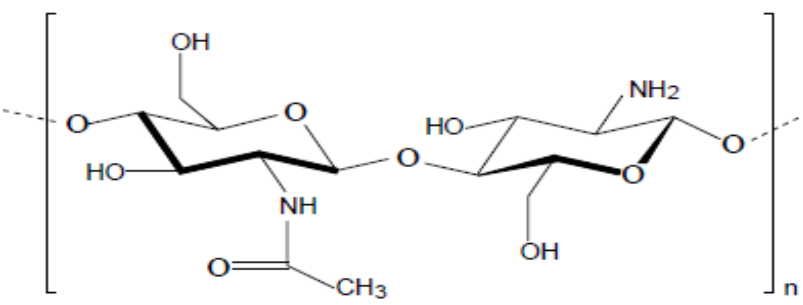

Figure 1. Chemical structure of chitosan consisting

of $\mathrm{N}$-acetyl-D-glucosamine and glucosamine units.

\section{Research Methods}

Natural polymers are widely used in the regenerative field of medicine, for wounds and burns dressing because of their unique properties such as biocompatibility, biodegradability and similarity to macromolecules recognized by the human body.

The selection of the natural polymers in this review was based on the complexing capacity with chitosan. The structural characteristics are presented, as well as the therapeutical effects and data on the biodegradability of these complexes in comparation with data from ohter reseaches and specialty literature.

\section{Results and Discussions}

Polyelectrolyte Complexes between Chitosan and Natural Polymers

\section{Chitosan-alginate polyelectrolyte complex}

Alginate is a natural, linear, unbranched, biodegradable polysaccharide and it consists of 1,4-linked $\beta$-D-mannuronic acid and $\alpha$-L-guluronic acid monomers in varying proportions (Figure 2). Alginates are extracted from brown seaweeds and marine algae such as Laminaria hyperborea, Ascophyllum nodosum and Macrocystis pyrifera $[8,9]$.

In order to form a polyelectrolyte complex, the negatively charged carboxylic acid groups of manuronic and guluronic acid units in alginate interact electrostatically with the positively charged amino groups of chitosan.

Due to the biodegradability and biocompatibility of the polyelectrolyte complex formed between these two polymers, alginate is one of the most studied anionic polyelectrolytes in complexation with chitosan. This polyelectrolyte complex is mechanically stronger at lower $\mathrm{pH}$ values where chitosan dissolves [10]. A study on the biodegradation of chitosan-alginate polyelectrolyte complexes showed that while chitosan alone, with a low degree of deacetylation, was effectively degraded by lysozymes, the effect of these enzymes on the polyelectrolyte complex was negligible. The ability of the polyelectrolyte complex of lysozyme absorbtion was high, but enzymatic degradation was hindered by the strong interaction between the chitosan and alginate polymeric chains.

Since it was demosntrated that the rate of biodegradation may be regulated by changing the polymer ratio, it indicates that this particular polyelectrolyte complex has a high potential in tissue engineering for scaffolds and support materials [11].

Chitosan-alginate polyelectrolyte complex fibers showed promising results for controlling the release of charged molecules and exhibited high encapsulation efficiencies of these molecules [12]. 


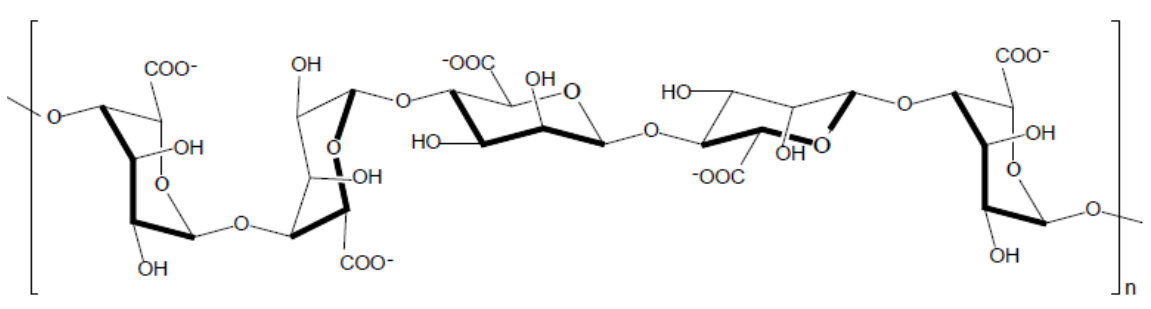

Figure 2. Chemical structure of alginate

\section{Chitosan-carrageenan polyelectrolyte complex}

Carrageenan is the generic name for a family of high molecular weight sulphated polysaccharides obtained from certain species of red seaweeds. There are three basic types of carrageenan, namely kappa $(\kappa)$, iota (I) and lambda $(\lambda)$ carrageenan (Figure 3) $[13,14]$.

a

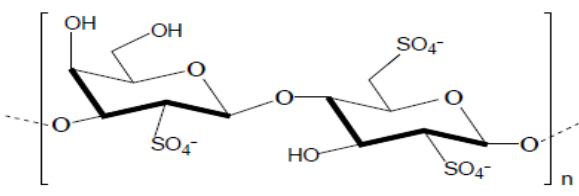

$\mathrm{b}$

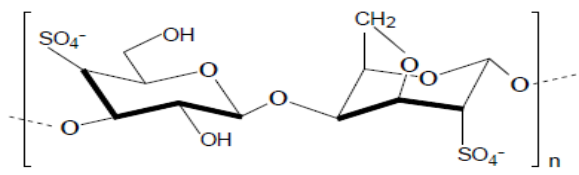

c

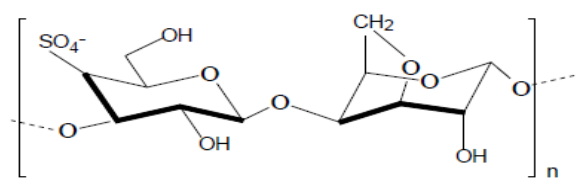

Figure 3. Chemical structures of (a) $\lambda$-carrageenan, (b) I-carrageenan and (c) K-carrageenan

Recent reports have shown that the nature or type of carrageenan considerably influence the characteristics of the polyelectrolyte complex that is formed with chitosan. The mechanical strength of polyelectrolyte complex gels formed between chitosan and different carrageenans were in the order $\lambda->1->\mathrm{K}$ - carrageenan. The gels obtained for $\mathrm{I}$ - and $\mathrm{k}$ carrageenan were temperature sensitive because of the helix-coil conformational transitions in their molecules [15].

3.

Chitosan-pectin polyelectrolyte complex

negatively charged carboxylic acid groups of pectin and the positively charged amino groups of chitosan can occurre. Furthermore, by adjusting the $\mathrm{pH}$ of this mixture a polyelectrolyte complex could be obtained. 


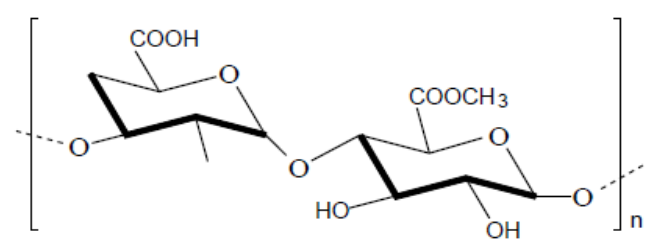

Figure 4. Chemical structure of pectin.

As expected, the extent of this interaction depended on the $\mathrm{pH}$ of the surrounding medium which determined the extent of ionization of the polymers [6].

\section{Chitosan-xanthan gum polyelectrolyte complex}

Xanthan gum is an exopolysaccharide secreted from Xanthomonas campestris.

It consists of $\beta$-(1,4)-D-glucopyranose glucan, a cellulosic backbone, and presents on every second glucose residue a trisaccharide side chain, namely (3,1)-a-D-mannopyranose-(2,1)- $\beta$-D-glucuronic acid-(4,1)- $\beta$-D-mannopyranose, (Figure 5 ) [18].

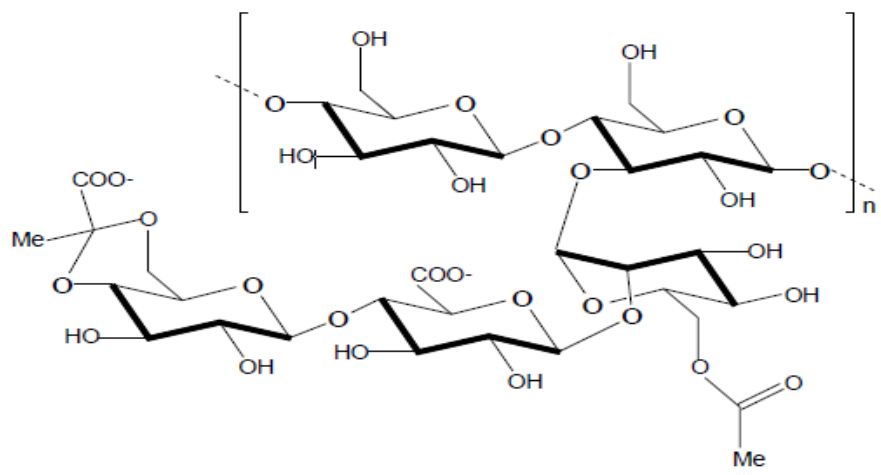

Figure 5. Chemical structure of xanthan gum

Results obtained from a modulated differential scanning calorimetry analysis and the swelling degree of microcapsules prepared from chitosan-xanthan gum polyelectrolyte complexes indicated that the cross-linking density was interdependent on xanthan concentration, chitosan concentration and chitosan solution $\mathrm{pH}$ [19].

\section{Chitosan-hyaluronic acid polyelectrolyte complex}

Hyaluronic acid or hyaluronan or hyaluronate is the only nonsulfated glycosaminoglycan found in the extracellular matrix throughout connective, epithelial and neural tissues.

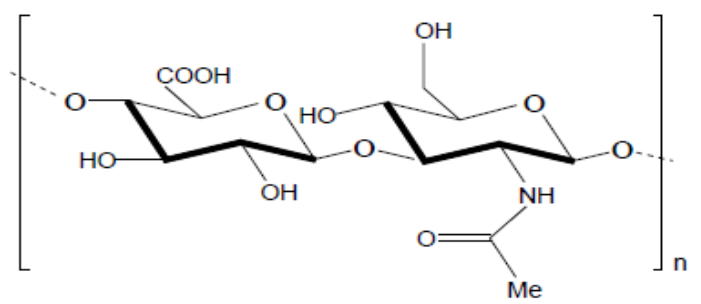

Figure 6. Chemical structure of hyaluronic acid. 
Hyaluronic acid is a linear anionic polysaccharide with high molecular weight that consists of $\beta-(1,3)-N$-acetyl-Dglucosamine and $a(1,4)$-D-glucuronic acid repeating units linked by $\beta(1 \rightarrow 3)$ bonds (Figure 6 ).

It is produced by bacterial fermentation of streptococcus species or by extraction processes from rooster combs, umbilical cords, synovial fluids or vitreous humour for commercial purposes. Hyaluronic acid has many applications in the medical field beeing used in ophthalmic surgery, arthritis treatment, in tissue engineering, a component of scaffolds for wound healing and implant devices $[2,20,21]$. Recent studies have shown that the polyelectrolyte complex between chitosan and hyaluronic acid protected hyaluronic acid against enzymatic hydrolysis, but only at $\mathrm{pH}$ values different from the optimal pH of the enzyme. The results from this studies revealed that the chitosan-hyaluronic acid polyelectrolyte complex unfortunately had less cell proliferation and wound healing effects compared to chitosan alone [22].

6.

Chitosan-gelatine polyelectrolyte complex

Gelatine is a heterogeneous mixture of protein fractions consisting of single or multi-stranded polypeptides (Figure 7). The process of obtaining gelatine is by partial hydrolysis of animal collagen derived from skin, white connective tissues and bones. There are two tipes og gelatine: type $A$ and type $B$. Type $A$ gelatine is derived from pig skin by using acid hydrolysis and type $B$ gelatine is obtained by alkaline hydrolysis of cattle hides and bones [23].

It was shown that the polyelectrolyte complex between chitosan and gelatine can only occur at a pH value above 4.7 and below 6.2. Above this value the net charge on gelatine type $B$ is negative. A pH value of 4.7 represents the isoelectric point of gelatin. Above a pH value of 6.2 chitosan start to precipitate out of solution. [24].

Natural polymers are involved in the repair of damaged tissues and in skin regeneration by Inducing and stimulating the wound healing process. Biomaterial hydrogels are engaged in the pharmaceutical and biomedical area, due to their threedimensional cross-linked polymeric networks that are soaked with water or biological fluids, and are used especially for wound management, tissue engineering, drug delivery, and organ transplant.

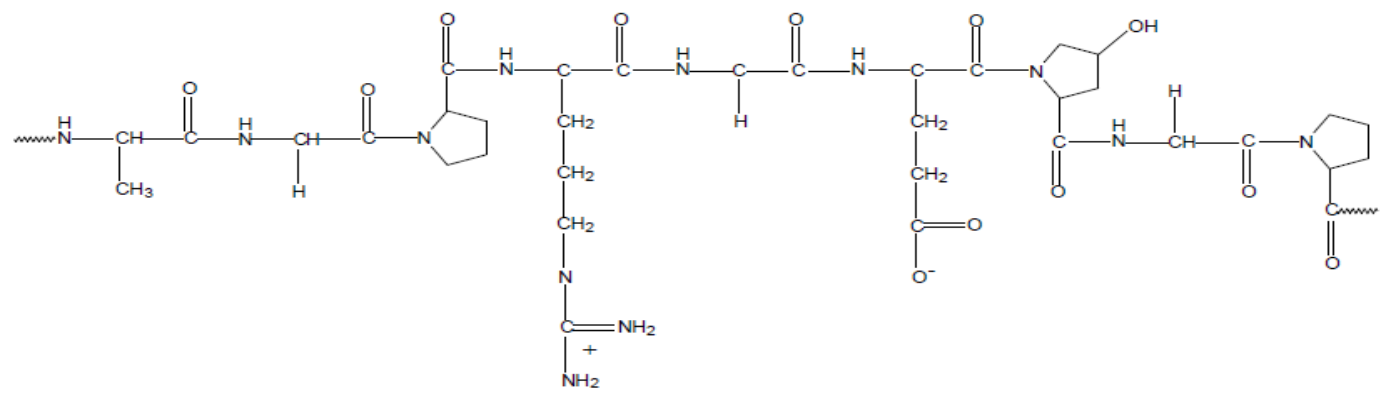

Figure 7. Typical structure of gelatin.

Hydrogels containing crosslinked natural polymers and polyelectrolyte complexes can be used for wounds and burns dressing.

\section{Conclusion}

Polyelectrolyte complexes combine unique physico-chemical properties of different polymers with the advantage of retaining high biocompatibility. It is therefore not surprising that polyelectrolyte complexes are gaining importance in modern pharmaceutical technology. In the management of wounds and burns dressings play an important role. The use of threedimensional polymeric scaffolds for cell targeting is already a common strategy for tissue engineering. Recent studies upon the properties of natural polymers (biocompatibility and biodegradability) will lead to a substantial development of novel types of wound dressings and to outstanding applications for regenerative medicine.

In the present, the most promising materials for wounds and burns dressing are still based on natural polymers such as polysaccharides (alginates, chitin, chitosan), proteoglycans and proteins (collagen, gelatin, fibrin, keratin, silk fibroin, eggshell membrane). 


\section{References}

[1] Josias H. Hamman, Chitosan Based Polyelectrolyte Complexes as Potential Carrier Materials in Drug Delivery Systems, Mar. Drugs 2010, 8, 1305-1322; doi:10.3390/md8041305

[2] Malafaya, P.B.; Silva, G.A.; Reis, R.L. Natural-origin polymers as carriers and scaffolds for biomolecules and cell delivery in tissue engineering applications. Adv. Drug Deliv. Rev. 2007, 59, 207-233.

[3] Caramella, C.; Ferrari, F.; Bonferoni, M.C.; Rossi, S.; Sandri, G. Chitosan and its derivatives as drug penetration enhancers. J. Drug Del. Sci. Tech. 2010, 20, 5-13.

[4] Berger, J.; Reist, M.; Mayer J.M.; Felt, O.; Gurny R. Structure and interactions in chitosan hydrogels formed by complexation or aggregation for biomedical applications. Eur. J. Pharm. Biopharm. 2004, 57, 35-52.

[5] Danielsen, S.; Strand, S.; de Lange Davies, C.; Stokke, B.T. Glycosaminoglycan destabilization of DNA-chitosan polyplexes for gene delivery depends on chitosan chain length and GAG properties. Biochim. Biophys. Acta 2005, $1721,44-54$

[6] Bernabe, P.; Peniche, C.; Argüelles-Monal, W. Swelling behavior of chitosan/pectin polyelectrolyte complex membranes. Effect of thermal cross-linking. Polym. Bull. 2005, 55, 367-375.

[7] Krayukhina, M.A.; Samoilova, N.A.; Yamskov, I.A. Polyelectrolyte complexes of chitosan, formation, properties and applications. Russ. Chem. Rev. 2008, 77, 799-813.

[8] Sankalia, M.G.; Mashru, R.C.; Sankalia, J.M.; Sutariya, V.B. Reversed chitosan-alginate polyelectrolyte complex for stability improvement of alpha-amylase: Optimization and physicochemical characterization. Eur. J. Pharm. Biopharm. 2007, 65, 215-232.

[9] Beneke, C.E.; Viljoen, A.M.; Hamman, J.H. Polymeric plant-derived excipients in drug delivery. Molecules 2009, 14, 2602-2620.

[10] Hein, S.; Wang, K.; Stevens, W.F.; Kjems, J. Chitosan composites for biomedical applications: status, challenges and perspectives. Mater. Sci. Technol. 2008, 24, 1053-1061.

[11] Li, X.; Xie, H.; Lin, J.; Xie, W.; Ma, X. Characterization and biodegradation of chitosan-alginate polyelectrolyte complexes. Polym. Degrad. Stab. 2009, 94, 1-6.

[12] Liao, I-C.; Wan, A.C.A.; Yim, E.K.F.; Leong, K.W. Controlled release from fibers of polyelectrolyte complexes. J. Control. Release 2005, 104, 347-358.

[13] Beneke, C.E.; Viljoen, A.M.; Hamman, J.H. Polymeric plant-derived excipients in drug delivery. Molecules 2009, $14,2602-2620$.

[14] Coviello, T.; Alhaique, F.; Dorigo, A.; Matricardi, P.; Grassi, M. Two galactomannans and scleroglucan as matrices for drug delivery: Preparation and release studies. Eur. J. Pharm. Biopharm. 2007, 66, 200-209.

[15] Shumilina, E.V; Shchipunov, Y.A. Chitosan-carrageenan gels. Colloid J. 2002, 64, 372-378.

[16] Fry, S.C. Primary cell wall metabolism, tracking the careers of wall polymers in living plant cells. New Phytol. 2004, $161,641-675$.

[17] Sriamornsak, P.; Thirawong, N.; Weerapol, Y.; Nunthanid, J.; Sungthongjeen, S. Swelling and erosion of pectin matrix tablets and their impact on drug release behavior. Eur. J. Pharm. Biopharm. 2007, 67,211-219.

[18] Mundargi, R.; Patil, S.A.; Aminabhavi, T.M. Evaluation of acrylamide-grafted-xanthan gum copolymer matrix tablets for oral controlled delivery of antihypertensive drugs. Carbohydr. Polym. 2007, 69, 130-141.

[19] Argin-Soysal, S.; Kofinas, P.; Lo, Y.M. Effect of complexation conditions on xanthan-chitosan polyelectrolyte complex gels. Food Hydrocol. 2009, 23, 202-209.

[20] Kim, S.J.; Shin, S.R.; Lee, S.M.; Kim, I.Y.; Kim, S.I. Thermal characteristics of polyelectrolyte complexes composed of chitosan and hyaluronic acid. J. Macromol. Sci. 2003, A40, 807-815 
[21] Rinaudo, M. Properties and degradation of selected polysaccharides: hyaluronan and chitosan. Corros. Eng. Sci. Technol. 2007, 42, 324-334.

[22] Denuziere, A.; Ferrier, D.; Damour, O.; Domard, A. Chitosan-chondroitin sulphate and chitosan-hyaluronate polyelectrolyte complexes: biological properties. Biomaterials 1998, 19, 1275-1285.

[23] Attama, A.A. Polyelectrolyte complexes of EudragitL30D-55 and gelatine: Antinociceptive activity of entrapped piroxicam. Drug Deliv. 2007, 14, 155-162.

[24] Yin, Y.; Li, Z.; Sun, Y. Yao, K. A preliminary study on chitosan/gelatine polyelectrolyte complex formation. J. Mater. Sci. (Letters) 2005, 40, 4649-4652. 